\title{
Propagation of Nonclassical Light through a Semiconductor Microcavity
}

\author{
O. Di Stefano, S. Savasta, and R. Girlanda ${ }^{1}$ ) \\ INFM and Dipartimento di Fisica della Materia e Tecnologie Fisiche Avanzate, \\ Università di Messina, Salita Sperone 31, I-98166 Messina, Italy
}

\begin{abstract}
We extend recently developed schemes for field quantization in absorbing dielectric media with local susceptibilities to dielectric systems described by a nonlocal susceptibility, in order to quantize the electromagnetic field in quantum wells embedded in planar semiconductor microcavities. As an application of the formalism, we analyze the effects of the propagation through a semiconductor microcavity on a continuous-mode squeezed coherent state. In particular, we calculate the variance of the field transmitted through the microcavity in the case of strong coupling regime for different detection schemes, as a function of the detuning, the exciton-photon coupling and the exciton homogeneous broadening.
\end{abstract}

The growth in experiments on quantum optical processes which take place inside material systems has stimulated the development of techniques for the quantization of the electromagnetic field in dispersive and absorbing dielectrics. The quantization of the electromagnetic field in absorptive dielectrics is considerably more complicated since the loss couples the field to a reservoir whose oscillators act as noise sources. A comprehensive treatment of electromagnetic fields in dispersive and absorbing dielectrics based on the Langevin forces has been given in [1]. Recently Ho Trung Dung et al. [2] presented a quantization scheme for dispersive and absorbing inhomogeneous media including the full three-dimensional nature of the electromagnetic field. A different general three-dimensional quantization scheme based on the construction of a unitary time evolution and an $S$-matrix formalism for scattering from lossy objects is due to Tip [3]. These quantization schemes for dispersive and absorbing linear dielectrics provide expressions which can be applied directly to such problems as the effects of propagation through absorbing dielectrics on light that initially displays nonclassical features [4], or to calculate the field vacuum fluctuations and hence the spontaneous emission of atoms embedded in dielectric systems [5]. All these quantization schemes have been developed for dielectric media with a local susceptibility. We have recently presented a quantization scheme for planar dielectric systems which are properly described by a nonlocal complex susceptibility [6]. Quantum wells (QWs) and superlattices are an important example of dielectrics driven by the field via a nonlocal susceptibility [7, 8]. Here we apply the formalism to cavity embedded QWs and to the analysis of propagation through a semiconductor microcavity of a continuous-mode squeezed coherent signal. The analysis of degradation and reshaping of nonclassical optical pulses propagating through a semiconductor microcavity (MC) is of great interest for the optical

\footnotetext{
1) e-mail: Raffaello.Girlanda@unime.it
} 
communication technology also due to the possibility of coherent controlling resonant excitations in these systems [9]. Following Ref. [6], we solve the second-order Fredholm integral equation with a quantum noise source term for the electric field operator propagating in a MC. Owing to space limitations, we give here only the quantized field expressions in vacuum outside a symmetric MC $(|z|>L)$. These expressions provide all the information needed for describing input and output quantum fields. The positive components of the electric field operator at normal incidence outside the MC are given by

$$
\begin{aligned}
& \hat{E}^{+}(z, \omega)=\left(\frac{\hbar \omega}{4 \pi \epsilon_{0} c \mathcal{A}}\right)^{\frac{1}{2}}\left[\hat{a}_{\mathrm{R}}(\omega) \mathrm{e}^{i k_{z} z}+\hat{b}_{\mathrm{L}}(\omega) \mathrm{e}^{-i k_{z} z}\right], \quad z \leq-L, \\
& \hat{E}^{+}(z, \omega)=\left(\frac{\hbar \omega}{4 \pi \epsilon_{0} c \mathcal{A}}\right)^{\frac{1}{2}}\left[\hat{a}_{\mathrm{L}}(\omega) \mathrm{e}^{-i k_{z} z}+\hat{b}_{\mathrm{R}}(\omega) \mathrm{e}^{i k_{z} z}\right], \quad z \geq L .
\end{aligned}
$$

In Eq. (1) $\hat{a}_{\mathrm{R}(\mathrm{L})}(\omega)$ are the input photon operators related to the field traveling (at normal incidence) towards the MC and obeying the usual bosonic commutation rules, while $\hat{b}_{\mathrm{R}(\mathrm{L})}(\omega)$ are the output operators related to the field escaping from the MC. They are given by

$$
\hat{b}_{\mathrm{L}(\mathrm{R})}(\omega)=A(\omega) \hat{F}(\omega)+R(\omega) \hat{a}_{\mathrm{R}(\mathrm{L})}(\omega)+T(\omega) \hat{a}_{\mathrm{L}(\mathrm{R})}(\omega),
$$

where the noise Langevin operators $\hat{F}(\omega)$ describe the degrees of freedom of the reservoir, they have zero expectation values and satisfy the following commutation relations:

$$
\left[\hat{F}(\omega), \hat{F}^{\dagger}\left(\omega^{\prime}\right)\right]=\delta\left(\omega-\omega^{\prime}\right), \quad\left[\hat{F}(\omega), \hat{F}\left(\omega^{\prime}\right)\right]=0 .
$$

The coefficients $R(\omega), T(\omega)$, and $A(\omega)$ are the reflection, transmission, and absorption coefficients, respectively, which can be obtained by the standard transfer-matrix method [8] including the effects of the QWs inside the MC and of the layered dielectric system.

We can now examine how the propagation through the MC influences the squeezing of an incident signal beam. We take the signal beam traveling rightwards to the MC as a continuous-mode squeezed coherent state, such that produced by a degenerate parametric amplifier pumped at frequency $2 \Omega,|R\rangle=\hat{D}([\alpha(\omega)]) \hat{S}\left(\left[\sigma, \phi_{\sigma}\right]\right)|0\rangle$, where $\hat{S}\left(\left[\sigma, \phi_{\sigma}\right]\right)$ is the continuous-mode squeezing operator, and $\hat{D}([\alpha(\omega)])$ is the displacement operator for the generation of continuous-mode coherent states $[4,10]$. We also will analyze the case in which a nonclassical input leftward signal is sent to the sample in addition to the rightward signal. We can choose for the state describing the input leftward a continuous-mode squeezed vacuum state $\left(|L\rangle=\hat{S}\left(\left[\sigma^{\prime}, \phi_{\sigma^{\prime}}\right]\right)|0\rangle\right)$. Quadrature squeezing occurs when the quantum fluctuations in one of the quadrature components of electromagnetic fields drop below the vacuum level, this is characterized by states of the field with no classical analogues. The effect of squeezing can be measured by a balanced homodyne detection scheme, see e.g. $[4,10]$. For a sufficiently long detection time, it is possible to obtain a simple expression of the variance of the homodyne measurements made on a specific field state, e.g. $|R\rangle$ as a function of the local oscillator (LO) frequency $\omega_{\mathrm{LO}}$ and phase $\phi_{\mathrm{LO}}[4]$,

$$
\left\langle\left[\Delta \hat{a}_{\mathrm{R}}\left(\phi_{\mathrm{LO}}, \omega_{\mathrm{LO}}\right)\right]^{2}\right\rangle-1=\left\langle\hat{a}_{\mathrm{R}}^{\dagger}\left(\omega_{\mathrm{LO}}\right), \hat{a}_{\mathrm{R}}\left(\omega_{\mathrm{LO}}\right)\right\rangle-\operatorname{Re}\left[\left\langle\hat{a}_{\mathrm{R}}^{\dagger}\left(\omega_{\mathrm{LO}}\right), \hat{a}_{\mathrm{R}}^{\dagger}\left(\omega_{\mathrm{LO}}\right)\right\rangle \mathrm{e}^{2 i \phi_{\mathrm{LO}}}\right],
$$

where we have used the standard notation $\langle\hat{a}, \hat{b}\rangle=\langle\hat{a} \hat{b}\rangle-\langle\hat{a}\rangle\langle\hat{b}\rangle$. The expectation values in (4) of course depend on the specific field state. For the above considered input 
rightward field, we obtain

$$
\begin{aligned}
& \left\langle\hat{a}_{\mathrm{R}}^{\dagger}(\omega), \hat{a}_{\mathrm{R}}\left(\omega^{\prime}\right)\right\rangle=\delta\left(\omega-\omega^{\prime}\right) \sinh ^{2}(\sigma(\omega)), \\
& \left\langle\hat{a}_{\mathrm{R}}^{\dagger}(\omega), \hat{a}_{\mathrm{R}}^{\dagger}\left(\omega^{\prime}\right)\right\rangle=\frac{1}{2} \delta\left(\omega+\omega^{\prime}-2 \Omega\right) \sinh (2 \sigma(\omega)) \mathrm{e}^{-i \phi_{\sigma}} .
\end{aligned}
$$

If the squeezing strength $\sigma$ is zero the variance of the vacuum or of coherent states is recovered. Analogous expressions are obtained for the leftward input field. By using Eqs. (2), (5), and (6), and assuming a zero temperature reservoir, the variance of the field transmitted through to the right of the MC can be written in the form

$$
\begin{aligned}
\left\langle\left[\Delta \hat{b}_{\mathrm{R}}\left(\phi_{\mathrm{LO}}, \omega_{\mathrm{LO}}\right)\right]^{2}\right\rangle-1= & |R|^{2}\left(\left\langle\left[\Delta \hat{a}_{\mathrm{L}}\left(\phi_{\mathrm{LO}}-\arg R\right), \omega_{\mathrm{LO}}\right]^{2}\right\rangle-1\right) \\
& +|T|^{2}\left(\left\langle\left[\Delta \hat{a}_{\mathrm{R}}\left(\phi_{\mathrm{LO}}-\arg T, \omega_{\mathrm{LO}}\right)\right]^{2}\right\rangle-1\right),
\end{aligned}
$$

with the coefficients evaluated at $\omega_{\mathrm{LO}}$. We now use these results to analyze the propagation of quadrature noise through a symmetric MC made of a $\lambda$ layer of refractive index $n_{\mathrm{c}}=3.4$ with one $\mathrm{QW}$ embedded inside with a radiative decay rate of the bare exciton amplitude $\Gamma_{0}=0.064 \mathrm{meV}$ and with the bare exciton energy $\omega_{0}=1.583 \mathrm{eV}$. The mirrors of the MC are two DBRs with 20 double layers of refractive index $n_{1}=2.95$, $n_{2}=3.32$. The squeezing strength $\sigma$ and phases $\phi_{\sigma}$ of the input rightward signal are taken to be constant over the resonance region. In particular we chose $\sigma=1$ and $\phi=2 \phi_{\mathrm{LO}}$ producing a variance of the homodyne measurements on the input field $\left\langle\left[\Delta \hat{a}_{\mathrm{R}}\left(\phi_{\mathrm{LO}}, \omega_{\mathrm{LO}}\right)\right]^{2}\right\rangle-1=-0.865$. No leftward input is sent. The transmitted variance as a function of the local oscillator frequency, for an MC resonant with the bare exciton energy $\omega_{0}$ and for different values of the exciton homogeneous broadening $\gamma$ is displayed in Fig. 1. The strong variations of the quadrature fluctuations as a function of $\omega_{\mathrm{LO}}$ are due to the large phase variations throughout the resonance region of the reflection and transmission coefficients. As it appears from Eq. (7), variance measurements are sensitive to the phase of the transmission and reflection coefficients in contrast to intensity measurements. The low level of output squeezing is due to the strong absorption in the resonance region and to the fact that a significant part of the signal is lost by reflection. The strong phase variations can be compensated by sending, before

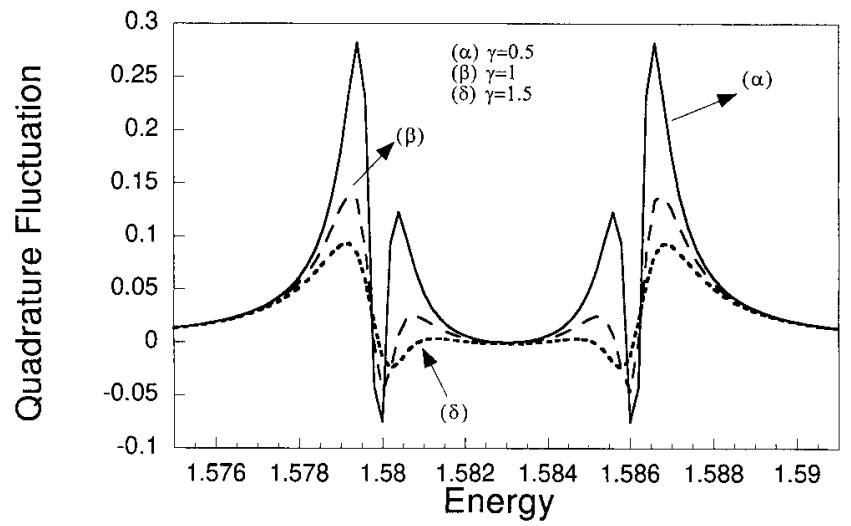

Fig. 1. Transmitted quadrature variance of Eq. (7) as the local oscillator frequency is swept through the Rabi peaks of a semiconductor microcavity for different values of the exciton homogeneous broadening $\gamma$ (in $\mathrm{meV}$ ). The input signal beam is a rightward propagating continuousmode squeezed state. Parameters are given in the text and in the figure (energy in eV) 


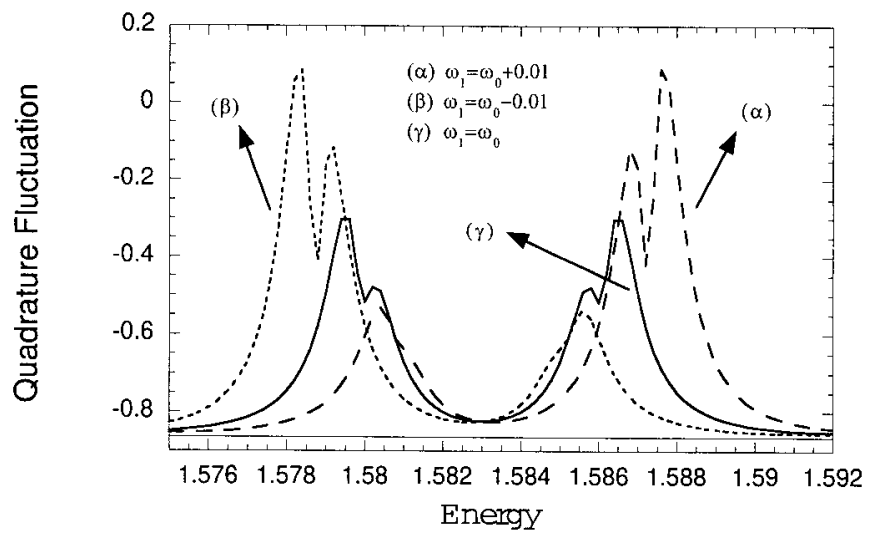

Fig. 2. Transmitted quadrature variance as the local oscillator frequency is swept through the Rabi peaks of a semiconductor microcavity for different values of microcavity detuning. The input signal beams are a rightward and a leftward propagating continuous-mode squeezed state. Parameters are given in the text and in the figure (energy in $\mathrm{eV}$ )

detection, also the local oscillator through the MC. The loss of squeezing by reflection can be compensated sending also an input leftward squeezed signal. We chose $\sigma^{\prime}=\sigma$ and $\phi^{\prime}=\phi$. The obtained quadrature fluctuations for different cavity detunings and for $\gamma=0.8 \mathrm{meV}$ are displayed in Fig. 2. As a reference we have also displayed the constant input noise level $\left\langle\left[\Delta \hat{a}_{\mathrm{R}}\right]^{2}\right\rangle-1=\left\langle\left[\Delta \hat{a}_{\mathrm{L}}\right]^{2}\right\rangle-1=-0.865$. Squeezing is degraded at frequencies close to the Rabi peaks mostly in correspondence with the exciton-like Rabi peak.

In conclusion, we have presented quantum optical input-output relations for cavity embedded QWs. The adopted quantization scheme allows us to analyze the linear propagation of nonclassical light in a very simple way without adding much more complications with respect to the well-known semiclassical theory of MC polaritons. We have applied our scheme to the analysis of linear propagation of continuous-mode squeezed light through a $\mathrm{MC}$ in the strong coupling regime. Output quadrature noise exhibits degradation of squeezing due to absorption and reflection and noticeably variations as a function of frequency due to variations of the phase of the MC reflection and transmission coefficients. The application to the analysis of propagation through a semiconductor MC of nonclassical optical pulses is under current development.

\section{References}

[1] R. Matloob, R. Loudon, S. M. Barnett, and J. Jeffers, Phys. Rev. A 52, 4823 (1995).

[2] Ho Trung Dung, L. Knöll, and D. G. Welsch, Phys. Rev. A 57, 3931 (1998).

[3] A. TiP, Phys. Rev. A 57, 4818 (1998).

[4] M. Artoni and R. Loudon, Phys. Rev. A 59, 2279 (1999).

[5] S. M. Barnett, B. Huttner, and R. Loudon, Phys. Rev. Lett. 68, 3698 (1992).

[6] O. Di Stefano, S. Savasta, and R. Girlanda, Phys. Rev. A 60, 1614 (1999).

[7] K. Chо, J. Phys. Soc. Jpn. 55, 4113 (1986).

[8] L. C. Andreani, Phys. Lett. A 192, 99 (1994).

[9] D. S. Citrin, 6th Internat. Meeting Optics of Excitons in Confined Systems, August 30 to September 2, 1999, Ascona (Switzerland).

[10] L. Mandel and E. Wolf, Optical Coherence and Quantum Optics, Cambridge University Press, Cambridge 1995.

M. O. Scully and M. S. ZubaIRY, Quantum Optics, Cambridge University Press, Cambridge 1997. 